\title{
Second-order direct analysis of steel structures made of tapered members
}

\author{
S.L. Chan ${ }^{\mathrm{a} *}$, S.W. Liu ${ }^{\mathrm{a}}$ and Y.P. Liu ${ }^{\mathrm{a}}$ \\ aDepartment of Civil and Environmental Engineering, The Hong Kong Polytechnic University \\ *corresponding author, e-mail address: ceslchan@polyu.edu.hk
}

\begin{abstract}
Mild steel hot-rolled sections are commonly prismatic because of the rolling process with a furnace, but welded sections made from steel plates do not have such a constraint, especially when robotic welding machines are used. The weight saving could be very significant by, say, using wide flanged section at mid-span and a small flanged section at ends of a simply supported beam. However, design codes do not provide formulae for buckling check of tapered members. This paper proposes a code-free second-order direct analysis for stabilty design of steel frames made of tapered members. The design is further applied to a single layered mega space frame of $136 \mathrm{~m}$ span. In the whole design process based on the concept of Second-order Direct Analysis, no uncertain effective length and independent member buckling checking are required.
\end{abstract}

Keywords: tapered members; stability design; buckling; steel frames

\section{Introduction}

Structural members with tapering sections can be fabricated quite easily by robotic welding of steel plates which are the common structural form of high strength steel of grade 500 or above. It also provides an aesthetic solution for singlelayered domes and long span structures. The structural form reduces significantly the weight of a structure.

Surprisingly, the use of tapered sections is unpopular, likely because little guidance is provided for design of structures made of tapered members. Nevertheless, the structural form is becoming popular in the reion of Hong Kong and Macau. Shown in Fig.1 is an example of a recently completed single-layer dome designed utilizing the tapering I-sections and without effective length.

An accurate second-order direct analysis based on nonlinear simulation technique is essential to the successful design of complex single-layer domes in which the snap-through buckling could occur under patterned loads or due to initial imperfections which is important for design and analysis of domes. Commonly, global imperfections are assumed in the mode as the first Eigen-buckling mode shape, which is recommended in codes and is the likely deformed mode shape in buckling. In addition to global buckling mode, the initial member curvatures are also important for a safe and reliable design of structures against buckling and several codes require their considerations (see Hong Kong steel code [1], Eurocode 3 [2] and AISC [3]).
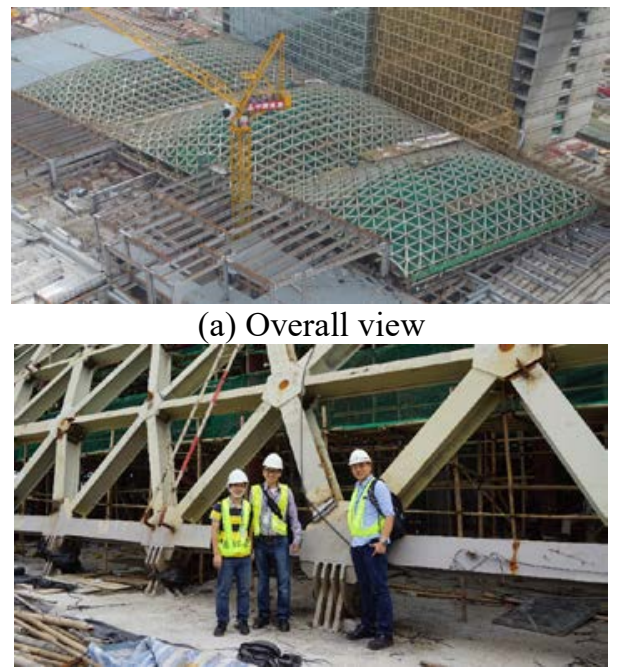

(b) Authors standing in front of the dome

Fig. 1. A complex single-layer dome consisting of members with tapered sections. 
The computer model using one element per member not only increases the numerical efficiency, but also removes the difficulty in modeling the member initial imperfections. Therefore, an advanced and robust element for large deflection and simulation of the initial curvature is crucial. Gu and Chan [4] developed the stability function element with the explicit consideration of member imperfections, and it is a closed-form solution and suitable for simulating the extremely slender members. Recently, Liu et al $[5,6]$ derived the curved Arbitrarily-Located-Hinge (ALH) element with an objective to capture the highly inelastic behaviors along the member length. The element tapering ALH is adopted here and used to design a practical structure which seems to be new and original in structural design technology.

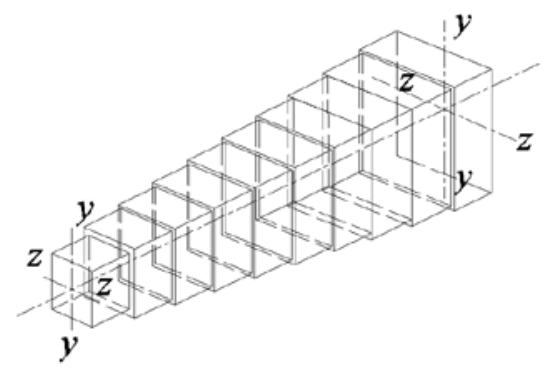

Fig. 2. Stepped elements representation method.

A tapering I-sections member is shown in Fig.2. To model the member with tapering sections, two methods are usually adopted as the approximated stepped elements and the tapering element approaches. The approximated stepped element method (ASEM) assumes the distribution of flexural rigidity along the member length as linear, parabolic or cubic in analysis and assigned manually and the errors can be large when using improper assumptions. This method is tedious and requires division of a member into many elements else the deflections and stiffness can be wrongly estimated. The other modeling approach is the exact tapering element method (ETEM) as shown in Fig.3. However, this method requires more element formulation efforts but reduces the computer efforts significantly. When using ASEM, at least twenty elements are required when using this technique for an accurate simulation. In the present study, the ETEM is used and an exact analytical approach by explicit modeling of the non-prismatic members by the tapering stiffness factors is proposed.
In addition to element formulation, a kinematic method is required to describe the motion and large deflection of a deforming element. The incremental tangent stiffness method proposed by Chan [7] is used and the equilibrium is established on the last configuration in the incremental-iterative procedure. This method is tested to be efficient and reliable. (see Chan [8, 9], Zhou and Chan [10].

\section{Assumptions}

In the present study, the following assumptions are adopted. (1) Small strains but large deformations are assumed and handled using the incremental tangent stiffness method; (2) Euler-Bernoulli hypothesis is adopted and the strain distribution along the cross section is linear; (3) Second-order effects including the P$\Delta$ and P- $\delta$ deformations are considered; (4) Loads are conservative and applied on element nodes and (5) Deformations due to the warping, shear and twist actions are not considered but they are supposed to be dealt with by additional checking equations in codes.

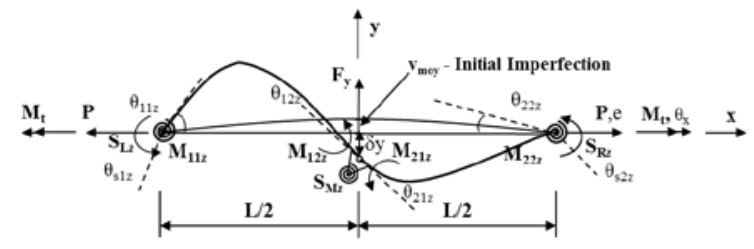

(a) Local x-y plan

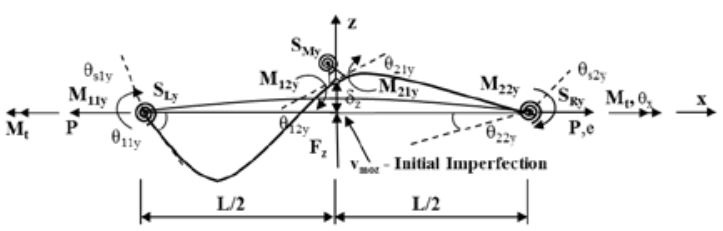

(b) Local x-z plan

Fig. 3. Forces versus displacements relations of the proposed element.

\section{Tapering beam-column element}

In the present study, the curved beam-column element with explicit consideration of the stiffness of tapering I-sections in element formulation is adopted. This element is derived from the arbitrarily-located-hinge (ALH) element proposed by Liu et. al $[5,6]$. The features of the proposed element includes: (1) High accuracy for the members with tapering Isections; (2) Member initial imperfection is explicitly considered; (3) Large deformations 
due to the existence of internal degree of freedoms are allowed; and (4) Second-order effects due to large deflection and axial compression are considered.

The co-rotational description is used and the member deflections are separated from the nodal translations so the element formulations can be simplified and concise.

Bending moments and axial loads dominate in an analysis. The axial and torsional deformations are relatively small in analysis. Here, the axial and torsional stiffness are calculated by averaging the values at the integration points along the member length, given as:

$$
\mathrm{A}=\frac{\sum A_{i}}{n} \quad \text { and } \mathrm{J}=\frac{\sum J_{i}}{n}
$$

where, $A$ and $J$ are the averaged values of the tapered sections; $\mathrm{n}$ is the number of the integration points and usually equals to twenty empirically; and $\mathrm{Ai}$ and $\mathrm{Ji}$ are the section area and the polar moment of inertia at the integration points along member length.

The above simplifications are valid for most structural members in practice. As stated in the assumptions, the consideration of lateraltorsional buckling is required for design of slender beams, which can be achieved by additional checking according to the empirical formulae in codes, e.g. Eurocode 3 [2].

\section{Element Shape Functions}

The basic forces versus displacements relations for the three-dimensional element are depicted in Fig. 3. The initial imperfections along two principle axes can be expressed as,

$$
\begin{aligned}
& \mathrm{v}_{0 \mathrm{y}}=\mathrm{v}_{\mathrm{m} 0 \mathrm{y}} \frac{\left(L^{2}-4 x^{2}\right)}{L^{2}} \\
& \mathrm{v}_{0 \mathrm{z}}=\mathrm{v}_{\mathrm{m} 0 \mathrm{z}} \frac{\left(L^{2}-4 x^{2}\right)}{L^{2}}
\end{aligned}
$$

where $v_{0 y}$ and $v_{0 z}$ are the lateral displacement functions of an imperfect element along $\mathrm{y}$-axis and $\mathrm{z}$-axis respectively; $\mathrm{v}_{\mathrm{moy}}$ and $\mathrm{v}_{\mathrm{m} 0 \mathrm{z}}$ are respectively the amplitudes of initial imperfection at mid-span along y-axis and zaxis; $L$ is the length of the member; and $\mathrm{x}$ is the distance along the element.

The lateral displacement functions for the three-dimensional element are given by,

$$
\begin{aligned}
& \mathrm{v}_{y}=\left\{\begin{array}{lll}
\left\{N_{11}\right. & N_{12} & \left.N_{13}\right\} \\
\left\{N_{21}\right. & N_{22} & \left.N_{23}\right\}
\end{array}\right\}\left\{\begin{array}{lllll}
\theta_{11 y} & \theta_{12 y} & \left.\theta_{21}\right\}^{T} & -\mathrm{L} / 2 \leq x \leq 0 \\
N_{22 y} & \left.\delta_{2}\right\}^{T} & 0 \leq x \leq L / 2
\end{array}\right. \\
& \mathrm{v}_{z}=\left\{\begin{array}{lll}
\left\{N_{11}\right. & N_{12} & N_{13}
\end{array}\right\} \cdot\left\{\begin{array}{lll}
\theta_{11 z} & \theta_{12 z} & \delta_{y}
\end{array}\right\}^{T},-\mathrm{L} / 2 \leq x \leq 0
\end{aligned}
$$

in which, $v_{y}$ and $v_{z}$ are the lateral displacement functions along $\mathrm{y}$-axis and $\mathrm{z}$-axis respectively; $\theta_{11 y}, \theta_{11 z}, \theta_{22 y}$ and $\theta_{22 z}$ are the external rotations at element ends and about two principle axes; $\theta_{12 y}, \theta_{12 z}, \theta_{21 y}$ and $\theta_{21 z}$ are the internal lateral deflections and rotations about the two principle axes; $\delta_{y}, \delta_{z}$ are the displacements along the two principle axes, and $\mathrm{N}_{11}, \mathrm{~N}_{12}, \mathrm{~N}_{13}, \mathrm{~N}_{21}, \mathrm{~N}_{22}$ and $\mathrm{N}_{23}$ are the shape functions given by:

$$
\begin{aligned}
& N_{11}=\frac{2 x^{2}(L+2 x)}{L^{2}} \\
& N_{12}=\frac{x(L+2 x)^{2}}{L^{2}} \\
& N_{13}=\frac{(L-4 x)(L+2 x)^{2}}{L^{3}} \\
& N_{21}=\frac{(L-2 x)^{2} x}{L^{2}} \\
& N_{22}=-\frac{2(L-2 x) x^{2}}{L^{2}} \\
& N_{23}=-\frac{(-L-4 x)(L-2 x)^{2}}{L^{3}}
\end{aligned}
$$

For the axial compression and lengthening, the respective displacement function can be conventionally assumed to be linear and the shape function can be determined as,

$$
\mathrm{u}=e\left(\frac{1}{2}+\frac{x}{L}\right)
$$

where $\mathrm{e}$ is the deformation along with axial force as illustrated in Fig. 3.

Similarly, the twist angle can be expressed by linear interpolation as,

$$
\mathrm{t}=\theta_{t}\left(\frac{1}{2}+\frac{x}{L}\right)
$$

where $\theta_{t}$ is the twist angle about the longitudinal $\mathrm{x}$-axis conjugate to the torsional moment.

\section{Bowing Effects}

In addition to the axial shortening or lengthening due to axial load, the distance between the two end nodes will be shortened due to curvature and bending moments. This bowing effect can be calculated as,

$$
u_{b}=\frac{1}{2} \int_{L}\left(\dot{v}_{y}{ }^{2}+2 v_{0 y} \dot{v_{y}}\right) d x
$$




$$
+\frac{1}{2} \int_{L}\left(\dot{v}_{z}^{2}+2 v_{0 z} \dot{v_{z}}\right) d x
$$

\section{Total Potential Energy Function}

The secant relations can be formulated by the principle of stationary potential energy, which is required in the force recovery under the incremental-iterative Newton-Raphson type of nonlinear analysis. The total potential energy function $\Pi$ is given by,

$$
\Pi=U-V
$$

where $\mathrm{U}$ is the strain energy and $\mathrm{V}$ is the external work done.

The strain energy function with negligence of the shear strain energy can be written as,

$$
\begin{aligned}
& U=\frac{1}{2} \int_{L} E A \dot{u}^{2} d x \\
& +\frac{1}{2} \int_{L} E I_{y}(x) \ddot{v}_{y}{ }^{2} d x+\frac{1}{2} \int_{L} E I_{z}(x) \ddot{v}_{z}{ }^{2} d x \\
& +\frac{1}{2} \int_{L} G J \dot{t}^{2} d x+\frac{1}{2} \int_{L} P\left(\dot{v}_{y}{ }^{2}+2 v_{0 y} \dot{v}_{y}\right) d x \\
& +\frac{1}{2} \int_{L} P\left(\dot{v}_{z}{ }^{2}+2 \dot{v}_{0 z} \dot{v}_{z}\right) d x+\int_{\theta_{m y}} S_{m y} d \theta+\int_{\theta_{m z}} S_{m z} d \theta
\end{aligned}
$$

where, EA is the axial stiffness constant; $\mathrm{EI}_{\mathrm{y}}$ and $\mathrm{EI}_{\mathrm{z}}$ are the flexural stiffness about $\mathrm{y}$ - and $\mathrm{z}$ axes respectively; GJ is the torsional stiffness; $\theta_{m y}, \theta_{m z}, \mathrm{~S}_{\mathrm{my}}$ and $\mathrm{S}_{\mathrm{mz}}$ are the hinge rotations and stiffness of the internal plastic hinge at respectively $\mathrm{y}$ - and z- axes written as,

$$
\begin{aligned}
& \theta_{m y}=\theta_{12 y}-\theta_{21 y} \\
& \theta_{m z}=\theta_{12 z}-\theta_{21 z} \\
& S_{m y}=E I_{y} \cdot R_{m y} \\
& S_{m z}=E I_{z} \cdot R_{m z}
\end{aligned}
$$

in which, $R_{m y}$ and $R_{m z}$ are the hinge stiffness connecting the two sub-elements.

In a design based on the second-order elastic analysis or the approach of first-plastic-hinge analysis, the internal hinge stiffness is set to be infinitely large, which indicates that no plastic strain energy is absorbed. However, an advanced analysis method by introducing the plastic fiber hinge approach proposed by Liu et. al $[5,6]$ to consider the material yielding is discussed in the followings.

In the present study, a rigorous consideration of the flexural stiffness distributions of the tapered I-sections along the member length is introduced and the second moments of inertia about both axes are defined as:

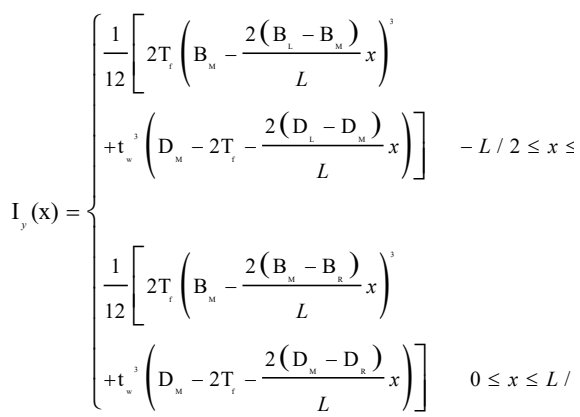

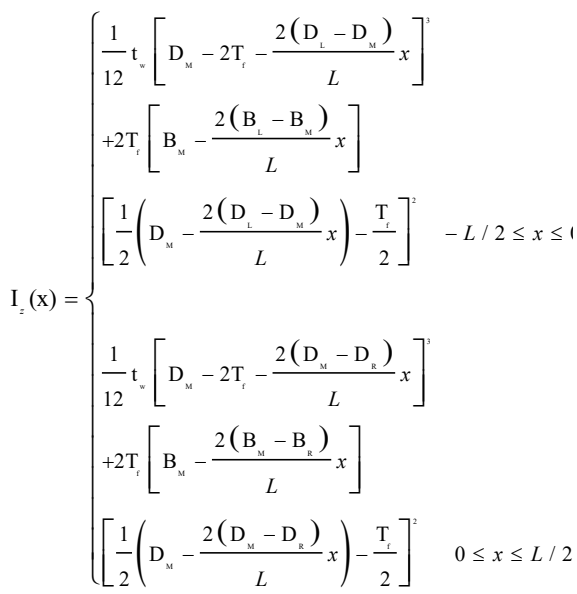

where, $B_{L}$ and $B_{R}$ are the overall width of the sections at the left and the right ends, respectively; $D_{L}$ and $D_{R}$ are the overall depth of the sections at the left and the right ends, respectively; $T_{f}$ and $t_{w}$ are respectively the plate thickness of the flange and the web; and the $\mathrm{B}_{\mathrm{m}}$ and $\mathrm{D}_{\mathrm{m}}$ are calculated as:

$$
\mathrm{B}_{\mathrm{M}}=\left(\mathrm{B}_{\mathrm{L}}+\mathrm{B}_{\mathrm{R}}\right) / 2 \quad \mathrm{D}_{\mathrm{M}}=\left(\mathrm{D}_{\mathrm{L}}+\mathrm{D}_{\mathrm{R}}\right) / 2
$$

The external work done can be expressed as,

$$
V=\sum F_{i} u_{i}
$$

\section{Tangent stiffness matrix}

In the incremental-iterative numerical procedure, the tangent stiffness is required to be formulated for the prediction of displacement increment due to an incremental load vector. The tangent stiffness matrix can be obtained by the second variation of the total potential energy function as,

$$
\begin{aligned}
& \delta^{2} \Pi=\frac{\partial^{2} \Pi}{\partial u_{i} \partial u_{j}} \delta u_{i} \delta u_{j}=\left[\frac{\partial F_{i}}{\partial u_{j}}+\frac{\partial F_{j}}{\partial P} \frac{\partial P}{\partial u_{j}}\right] \delta u_{i} \delta u_{j} \\
& \text { and } \mathrm{i}, \mathrm{j}=1 \sim 12
\end{aligned}
$$


where $F_{i}$ and $u_{i}$ are the force and displacement vectors respectively. Rearranging, the tangent stiffness of the element can be written in three components as,

$$
\left[k_{e}\right]=\left[k_{L}\right]+\left[k_{G}\right]+\left[k_{S}\right]
$$

in which, $\left[k_{e}\right]$ is the tangent stiffness of the element; $\left[k_{L}\right]$ is the linear stiffness matrix as:

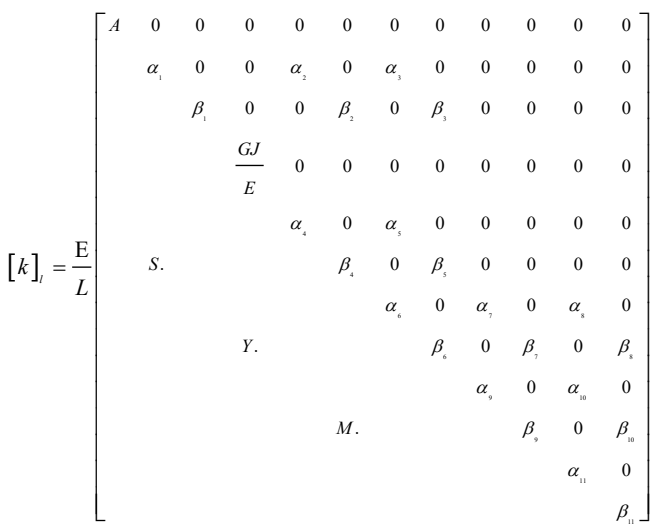

$\left[k_{G}\right]$ is the geometric stiffness matrix; and $\left[k_{S}\right]$ is the spring stiffness for the internal hinge and can be found in the paper by Liu et. al $[5,6]$.

In order to incorporate the proposed element into the existing program efficiently, the degrees of freedom (DOF) of the internal nodes $\left\{\mathrm{u}_{i}\right\}$ will be condensed. The internal and external DOFs and the related forces can be expressed as,

$$
\begin{aligned}
& \left\{u_{i}\right\}=\left\{\begin{array}{llllll}
\theta_{12 y} & \theta_{12 z} & \delta_{z} & \delta_{y} & \theta_{21 y} & \theta_{21 z}
\end{array}\right\}^{T} \\
& \left\{u_{e}\right\}=\left\{\begin{array}{llllll}
e & \theta_{11 \mathrm{y}} & \theta_{11 \mathrm{z}} & \theta_{\mathrm{x}} & \theta_{22 \mathrm{y}} & \theta_{22 \mathrm{z}}
\end{array}\right\}^{T} \\
& \left\{F_{i}\right\}=\left\{\begin{array}{llllll}
M_{12 y} & M_{12 z} & F_{z} & F_{y} & M_{21 y} & \left.M_{21 z}\right\}^{T}
\end{array}\right. \\
& \left\{F_{e}\right\}=\left\{\begin{array}{lllllll}
P & M_{11 y} & M_{11 z} & M_{x} & M_{22 y} & M_{22 z}
\end{array}\right\}^{T}
\end{aligned}
$$

The condensed stiffness $\left[\mathrm{k}^{*}\right]$ and generalized force $\{f\}$ can be expressed as,

$$
\left[k^{*}\right]\left\{u_{e}\right\}=\{f\}
$$

in which,

$$
\begin{aligned}
& {\left[k^{*}\right]} \\
& =\left[k_{e e}\right]-\left[k_{i e}\right]^{T}\left[k_{i i}\right]^{-1}\left[k_{i e}\right] \\
& \{f\}=\left\{F_{e}\right\}-\left[k_{i e}\right]^{T}\left[k_{i i}\right]^{-1}\left\{F_{i}\right\}
\end{aligned}
$$

\section{Descriptions on Kinematic Motions}

To consider large deflections in a spatial analysis, the incremental secant stiffness method is adopted in conjunction with the updated Lagrangian description proposed by Chan [7] discussed below.

\section{The Incremental Secant Stiffness Method}

The incremental secant stiffness method is similar to its total incremental secant counterpart, where the equilibrium conditions are established with reference to the last-known configuration in the former approach as the original configuration in the latter method. However, the incremental rotations in each step should be limited to be small such that the rotation can be treated as moderately large and the infinitesimal rotations can be taken as vectorial quantities, but this is a natural constraint to a non-linear analysis requiring moderate incremental load steps. Further, the convergence rate of the incremental secant stiffness method is remarkably faster than the total secant stiffness approach.

This method has been successfully adopted by numerous researchers and reported to be efficient and effective for large deflection and inelastic analysis. Chan [7] used the incremental secant stiffness method for inelastic postbuckling analysis of the tubular member and found to achieve a stable convergence and efficiency in the numerical iterative procedure. Earlier, Yang and Chiou [11] utilized this approach for the large deflection of the planer frame and Argyris et al. [12] applied this method to the three-dimensional frame with large member rotations.

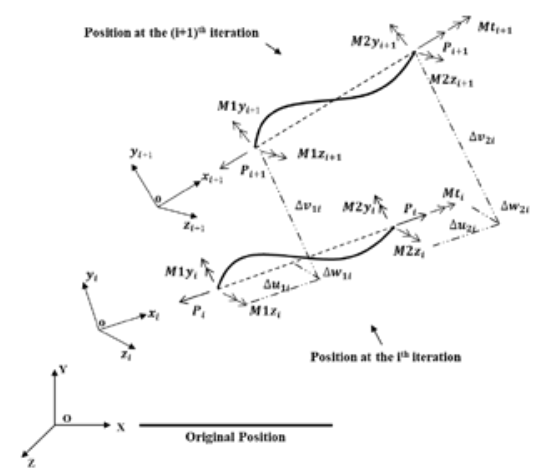

Fig. 4. Incremental kinematics of an element in three-dimensional space.

Therefore, the goal of the analysis at each load-increment is to find the resisting forces at the $i+1^{\text {th }}$ position by referring to the last-known configuration at the $i^{\text {th }}$ position Fig. 4. The natural incremental rotations in an element can be calculated as, 


$$
\begin{aligned}
\Delta \theta_{y 1 i} & =\Delta \alpha_{y 1 i}+\Delta \beta_{y i} \\
\Delta \theta_{y 2 i} & =\Delta \alpha_{y 2 i}+\Delta \beta_{y i} \\
\Delta \theta_{z 1 i} & =\Delta \alpha_{z 1 i}-\Delta \beta_{z i} \\
\Delta \theta_{z 2 i} & =\Delta \alpha_{z 2 i}-\Delta \beta_{z i}
\end{aligned}
$$

in which, $\Delta \alpha_{y 1 i}, \Delta \alpha_{y 2 i}, \Delta \alpha_{z 1 i}$ and $\Delta \alpha_{z 2 i}$ are the incremental rotations about the last known configuration; and $\Delta \beta_{y i}$ and $\Delta \beta_{z i}$ are the incremental rigid body rotations given by,

$$
\begin{aligned}
\Delta \beta_{y i} & =\frac{\Delta w_{2 i}-\Delta w_{1 i}}{L_{i}} \\
\Delta \beta_{z i} & =\frac{\Delta v_{2 i}-\Delta v_{1 i}}{L_{i}}
\end{aligned}
$$

where, $L_{i}$ is the member length at the last known configuration; $\Delta w_{1 i}, \Delta w_{2 i}, \Delta v_{1 i}$ and $\Delta v_{2 i}$ are the displacements at member along local z- and y-axes respectively.

The relative incremental twist about the shear center can be simply evaluated as,

$$
\Delta \theta_{x i}=\Delta \theta_{x 2 i}-\Delta \theta_{x 1 i}
$$

The incremental axial lengthening can be determined as,

$$
\begin{aligned}
& \Delta e_{i}=\Delta \mathrm{u}_{\mathrm{b}}-\Delta u_{n i} \\
& \text { where, } \\
& \Delta u_{n i}=\mathrm{L}_{i+1}-\mathrm{L}_{i}
\end{aligned}
$$

and the $\Delta u_{b}$ can be obtained by the first deviation of the expressions of $u_{b}$.

The consideration of the incremental bowing effect is vital for accuracy when using one element per member, which is usually ignored by some researchers and more elements are therefore needed to minimize the error. The comparison results of the including and excluding this incremental bowing effect has been reported by Chan [13].

Once the natural deformations are obtained, the incremental forces and moments can be evaluated as,

$$
\begin{aligned}
\left\{\Delta \mathrm{R}_{i}\right\} & =[K e]_{i}\left\{\Delta u_{i}\right\} \\
\left\{\mathrm{R}_{i+1}\right\} & =\left\{\mathrm{R}_{i}\right\}+\left\{\Delta \mathrm{R}_{i}\right\}
\end{aligned}
$$

where, $[\mathrm{Ke}]_{i}$ is the element stiffness matrix at the $\mathrm{i}^{\text {th }}$ configuration; and $\{\mathrm{R}\}$ is the internal resisting forces.

\section{Verification Examples}

To verify the accuracy, efficiency and versatility of the present method for the proposed design approach, several examples are selected and compared with the results by the conventional analysis method using stepped element model.

\section{Example 1: Extensive verifications on the single element}

In this example, tests on the single element under various types of loading conditions are conducted to validate the element performance on element number convergence. A nonprismatic member with tapered I-sections is chosen for the present study. The overall width and depth of the member vary from $500 \mathrm{~mm}$ to $1000 \mathrm{~mm}$ and $1000 \mathrm{~mm}$ to $500 \mathrm{~mm}$, respectively. The plate thickness at the flange and web are $30 \mathrm{~mm}$ and $25 \mathrm{~mm}$, respectively. The member length is 20 meter and it is simply supported. The Young's modulus and Poisson's ratio are $205000 \mathrm{MPa}$ and 0.3 , respectively. As aforementioned, the tapered sections stiffness factors can be calculated before the numerical incremental-iterative procedure.

The member will be analyzed by one of the proposed beam-column element under the following cases:

Case a: Pure bending about the major axis

Case b: Pure bending about the minor axis

Case c: Pure bending about both the axes

Case d: Uniaxial eccentric compression about the major axis

Case e: Uniaxial eccentric compression about the major axis

Case f: Bi-axial compression about both the axes

The conventional approach using stepped elements representation is adopted for the comparisons, where three types of modeling approaches selected as 5, 10 and 30 stepped elements per members. Herein, the analysis results from the model using 30 stepped elements are selected as the benchmarking solutions. The comparison results are plotted in Fig. 5 (a) to Fig. 5 (f).

From the comparisons in Table 1, the proposed element improves efficiency and accuracy for a tapered beam-column. The results from the analysis model using one TTH element per member are closed to those from the model using 30 stepped elements, where the averaged difference is only $1.26 \%$. However, the results from the models using 5 and 10 stepped elements per member are observed to have $18.56 \%$ and 
$5.34 \%$ discrepancy with the benchmark solutions.

As illustrated in the load versus deflections curves, the member deflections under different load conditions can be traced and predicted very well by the model using only one proposed beam-column element. The example indicates the numerical inefficient conventional stepped element representation can be replaced by the present element with numerical efficiency dramatically improved.

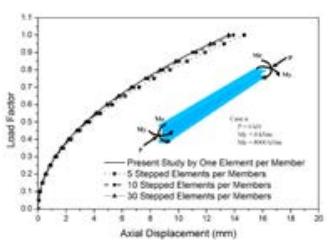

(a) Pure bending about the major axis

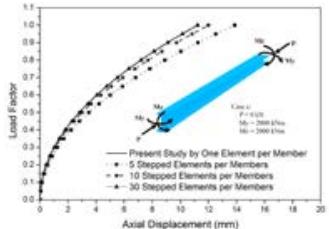

(c) Pure bending about both axes

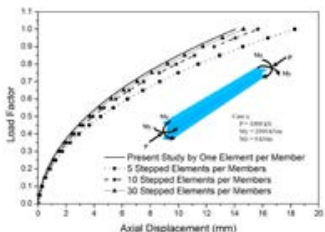

(e) Uniaxial compression about the minor axis

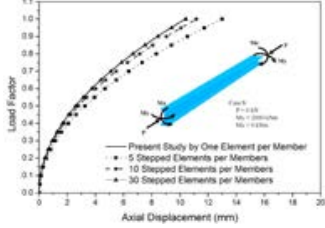

(b) Pure bending about the minor axis

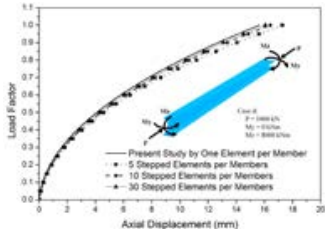

(d) Uniaxial compression about the major axis

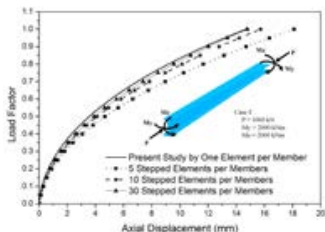

(f) Bi-axial compression about both axes
Fig. 5. Extensive verifications of the proposed element.

\section{Example 2: Second-order analysis of single cantilever columns with tapered I- sections}

This example tests the kinematics of large deflections of a single element and a cantilever element under bi-axial compression about both axes is analyzed. The column is non-prismatic with tapered I-sections, where the overall width and depth vary from $400 \mathrm{~mm}$ to $200 \mathrm{~mm}$ and 400 $\mathrm{mm}$ to $200 \mathrm{~mm}$, respectively. The plate thickness at the flange and web are both $30 \mathrm{~mm}$. The member length is $10 \mathrm{~m}$. The Young's modulus and Poisson's ratio are $205000 \mathrm{MPa}$ and 0.3, respectively.
The analysis results from the model using 30 stepped elements per member are adopted for the comparisons and presented in Fig. 6. From the comparisons, the loads versus deflections curves from the two models are very closed and nearly identical, and this further confirms the accuracy of the proposed element. Therefore, the conventional modeling method using stepped element representations can be improved significantly by adopting the proposed approach.

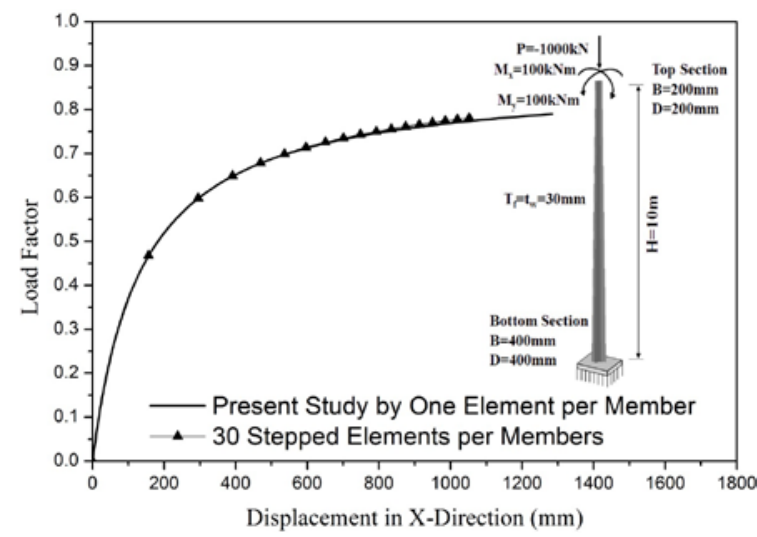

Fig. 6. Displacement in X-direction at top of the cantilever column.

\section{Example 3: Second-order analysis of a dome-like structure}

This example is to carry out a second-order analysis of a dome-like structure, where the geometry and loads are plotted in Fig. 7. The dome is vertically supported on several nodes with lowest attitude. The section assignments for the dome are given in Fig. 7, where the main arc members are tapered with I-sections and the secondary beams are uniform sections. The section dimensions and ID are given in Table 2. The Young's modulus and Poisson's ratio are $205000 \mathrm{MPa}$ and 0.3 , respectively.

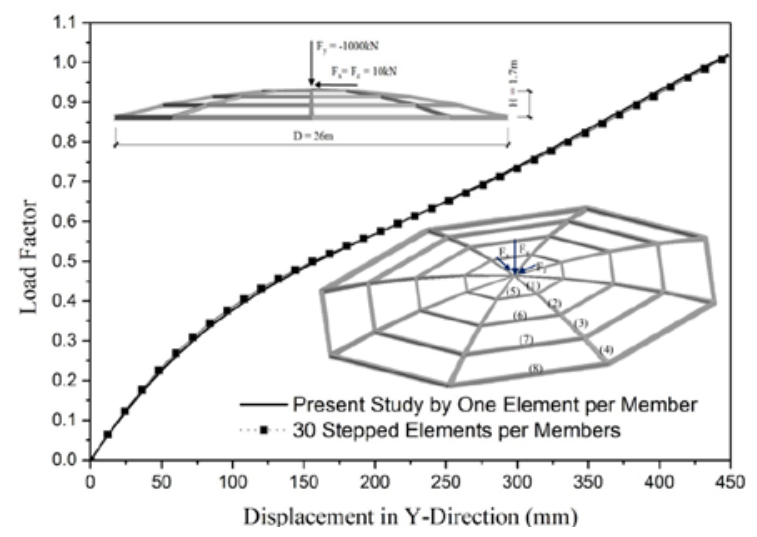

Fig. 7. Load versus displacement of the dome. 
The numerical model using 30 stepped elements for simulating the tapered members is introduced for the comparisons and the results are plotted in Fig. 7. The vertical displacements in Y-direction of the topmost node from the two models are plotted. The comparisons validate the feasibility of the proposed numerical model for second-order analysis of the dome structures, where one element per member model is used with high numerical efficiency.

\section{Conclusions}

In this paper, a curved beam-column element, named as the Tapered-Three-Hinges (TTH) element, is proposed for the applications in dome structures. To improve the numerical efficiency for models with approximated or stepped elements representations for simulating the members with tapered sections, an analytical and exact solution for consideration of the stiffness of tapered sections is developed via the use of a series of stiffness factors. To consider large deflection effects in analysis, the incremental secant stiffness method is introduced where the equilibrium is established by referring to the configuration in the last iteration. Extensive verifications are conducted for the proposed element, and the comparisons demonstrate the present approach in highly accurate and efficient, with the results by one proposed element close to 30 stepped elements by linearized stepped model.

\section{Acknowledgements}

The authors are grateful to the financial supports by the Research Grant Council of the Hong Kong SAR Government on the project "Second-Order Analysis of Shallow Dome Structures Made of Tapering Members (PolyU 152047/17E)" and "Second-Order Analysis of Flexible Steel Cable Nets Supporting Debris (PolyU 152008/15E)" and support by Innovation and Technology Fund of the Hong Kong SAR Government for the project "Development of an energy absorbing device for flexible rock-fall barriers (ITS/059/16FP)" and by the Hong Kong Branch of Chinese National Engineering Research Centre for Steel Construction of The Innovation and Technology Fund of the Hong Kong SAR Government for the project "Advanced Numerical Analysis for Building Structures using High Performance Steel Materials".

\section{References}

[1] Building Department of Hong Kong. Code of Practice for the structural use of steel. Hong Kong; 2011.

[2] The European Committee for Standardization (CEN). EN 1993-1-1: Eurocode 3: Design of Steel Structures. Part 1-1: General Rules and Rules for Buildings, Comité Europeo de Normalización; 2005.

[3] AISC. Steel Construction Manual; American Institute of Steel Construction; 2011.

[4] Chan SL, Gu JX. Exact tangent stiffness for imperfect beam-column members. Journal of Structural Engineering 2000; 126(9):1094-1102.

[5] Liu SW, Liu YP, Chan, SL. Advanced analysis of hybrid steel and concrete frames: Part 2: Refined plastic hinge and advanced analysis. Journal of Constructional Steel Research 2012; 70:337-349.

[6] Liu SW, Liu YP, Chan SL. Direct analysis by an arbitrarily-located-plastic-hinge element-Part 1: Planar analysis. Journal of Constructional Steel Research 2014; 103:303-315.

[7] Chan SL. Large Deflection Kinematic Formulations for 3-Dimensional Framed Structures. Computer Methods in Applied Mechanics and Engineering 1992; 95(1):17-36.

[8] Chan SL. Non-linear behavior and design of steel structures. Journal of Constructional Steel Research 2001; 57(12):1217-1231.

[9] Chan SL, Huang HY, Fang LX. Advanced analysis of imperfect portal frames with semirigid base connections. Journal of Engineering Mechanics 2005; 131(6):633-640.

[10]Zhou ZH, Chan SL. Self-equilibrating element for second-order analysis of semirigid jointed frames. Journal of engineering mechanics 1995; 121(8):896-902.

[11] Yang YB, Chiou, HT. Rigid body motion test for nonlinear analysis with beam elements. Journal of engineering mechanics 1987; 113(9): 14041419.

[12] Argyris J. An excursion into large rotations. Computer Methods in Applied Mechanics and Engineering 1982; 32(1):85-155.

[13] Chan SL, Zhou ZH. On the development of a robust element for second-order non-linear integrated design and analysis. Journal of Constructional Steel Research 1998; 47(1):169190. 